\title{
Jogos educacionais digitais para idosos: uma revisão sistemática de literatura
}

\author{
Ronan L. R. Ferreira ${ }^{1}$, Lucila Ishitani ${ }^{1}$ \\ ${ }^{1}$ Programa de Pós-graduação em Informática \\ Pontifícia Universidade Católica de Minas Gerais (PUC Minas) \\ Anel Rodoviário Km 23,5 - Rua Walter Ianni, 255 - São Gabriel - 31980-110 \\ Belo Horizonte - MG - Brasil \\ ronan.loschi@gmail.com, lucila@pucminas.br
}

\begin{abstract}
This paper presents the results of a systematic literature review focused on educational digital games for the elderly. The research questions were three: What are the cognitive limitations to consider when developing educational digital games for the elderly? Which are the topics of interest and the expectations of the elderly regarding educational digital games? And what are the contributions of educational digital games for the learning process of the elderly? The selected studies were classified according to the desired quality and contributed to partially answer the research questions. Furthermore, the use of the systematic literature review method confirmed the lack of publications in this area.
\end{abstract}

Resumo. Este artigo apresenta os resultados de uma revisão sistemática de literatura com foco em pesquisas sobre jogos digitais educacionais para idosos. As questões de pesquisa foram três: Quais as limitações cognitivas a serem consideradas no desenvolvimento de jogos digitais educacionais para idosos? Quais os temas de interesse e expectativas dos idosos com relação a jogos digitais educacionais? E quais as contribuições dos jogos digitais educacionais para o processo de aprendizagem dos idosos? Os estudos selecionados foram classificados quanto à qualidade pretendida e contribuíram para responder parcialmente às questões de pesquisa. Além disso, o uso do método de revisão sistemática de literatura permitiu confirmar a carência de publicações na área.

\section{Introdução}

Para [Chen et al. 2012a], jogos digitais são um elemento tecnológico que pode contribuir para a melhoria do processo de envelhecimento das pessoas, fornecendo subsídios para uma melhor qualidade de vida, pois podem amenizar as restrições decorrentes da idade, que atingem principalmente aspectos motores, perceptivos, cognitivos e psicossociais das pessoas.

Segundo a classificação da Organização Mundial de Saúde (OMS) [World Health Organization 2014], são considerados idosos os adultos com mais de 65 anos em países desenvolvidos e com mais de 60 anos em países subdesenvolvidos. De acordo com o relatório World Population Prospects - The 2012 Revision elaborado pela Nações Unidas, nas regiões mais desenvolvidas, a população com 60 anos ou mais terá um aumento de 45\%, passando de 287 milhões em 2013 para 417 milhões em 2050. 
Nas regiões menos desenvolvidas o número de indivíduos deve subir de 554 milhões em 2013 para 1,6 bilhões em 2050 [United Nations 2013]. No Brasil, a expectativa de vida era de 69,8 anos em 2013 e subiu para 74,9 anos [IBGE 2014]. Estima-se que em 2020, essa população poderá ultrapassar a quantidade de 30 milhões de pessoas [IBGE 2014]. Portanto, em um contexto em que nos deparamos com o aumento da expectativa de vida, torna-se importante identificar as pesquisas que estão sendo realizadas para ofertar aos idosos opções como os jogos educacionais para continuar aprendendo.

A revisão sistemática de literatura era um método já bastante conhecido em outras áreas de pesquisa, como a da saúde, e foi introduzida, na Computação, por [Kitchenham 2004]. Em seu trabalho, Kitchenham afirma que uma revisão sistemática de literatura é um meio de avaliar e interpretar os dados relevantes, disponíveis para a investigação de uma determinada questão de pesquisa, área, tópico, ou fenômeno de interesse. As revisões sistemáticas de literatura visam a apresentar uma avaliação justa de um tema de pesquisa, utilizando uma metodologia confiável, rigorosa e aditável.

Assim, o objetivo geral deste estudo foi aplicar o método da revisão sistemática de literatura para identificar pesquisas sobre jogos digitais educacionais para idosos. Para isso, seguiu-se o método da revisão sistemática de literatura com base em trabalhos relacionados de Kitchenham. O método da revisão sistemática de literatura é dividido em três fases: planejamento, execução e relatório. Seguindo esse método definiram-se três questões de pesquisa (Research Questions - RQ). Com a primeira questão buscou-se identificar quais as limitações cognitivas a serem consideradas no desenvolvimento de jogos digitais educacionais para idosos. Com a segunda questão buscou-se determinar quais os temas de interesse e expectativas dos idosos com relação a jogos digitais educacionais e já com a terceira questão buscou-se descobrir quais as contribuições dos jogos digitais educacionais para processo de aprendizagem dos idosos. Apenas 18 artigos foram selecionados e nenhum deles estava diretamente ligado ao tema da pesquisa, indicando a necessidade de mais pesquisas na área.

Este artigo está estruturado da seguinte forma: a Seção 2 descreve o método da pesquisa desenvolvido para a elaboração deste trabalho; a Seção 3 exibe os resultados da coleta de dados; a Seção 4 apresenta uma discussão dos resultados obtidos e, por fim, a Seção 5 apresenta as considerações finais deste trabalho.

\section{Fase 1 - Planejamento}

Segundo [Kitchenham 2004], as questões de pesquisa (Research Questions - RQ) definem o foco da revisão sistemática e são definidas na fase 1 do método. Além, disso, a especificação das questões de pesquisa é a parte mais importante de qualquer revisão sistemática, pois as questões irão conduzir toda a metodologia do processo de busca, extração dos dados (critérios de inclusão e exclusão), avaliação da qualidade dos dados e a própria busca pelas respostas das questões. As questões de pesquisa definidas para este estudo foram:

RQ1. Quais as limitações cognitivas a serem consideradas no desenvolvimento de jogos digitais educacionais para idosos?

RQ2. Quais os temas de interesse e expectativas dos idosos com relação a jogos digitais educacionais? 
RQ3. Quais as contribuições dos jogos digitais educacionais para o processo de aprendizagem dos idosos?

\subsection{Processo da busca}

Definidas as questões, deu-se início ao processo para definir o protocolo de revisão. Um protocolo pré-definido é necessário para reduzir a possibilidade de viés do pesquisador, como por exemplo, a seleção de estudos individuais e análises determinadas pelas expectativas do pesquisador.

O protocolo desenvolvido nesta pesquisa seguiu as diretrizes propostas por [Kitchenham 2004]. O protocolo foi verificado por ambos os autores deste trabalho. O protocolo foi composto de: estratégias da pesquisa, filtros, critérios de seleção, critérios de verificação da qualidade dos estudos, estratégia de extração dos dados, síntese dos dados extraídos, estratégia de divulgação.

\subsection{Estratégias da pesquisa}

A estratégia do processo de pesquisa foi uma busca por artigos publicados no período de 2004 a 2014, em bases de dados e em anais de conferências selecionada. Iniciouse o processo com a pesquisa eletrônica nas principais bases de dados pelos seguintes termos de pesquisa: educacional, aprendizagem, treinamento, idoso, pessoas idosas e jogo. A busca pelas bases foi feita prioritariamente pelo portal de periódicos da CAPES via CAPES CAFÉ - RNP e eventualmente pelo Google acadêmico.

As fontes de pesquisa selecionadas foram as seguintes: bibliotecas digitais da Association for Computing Machinery Digital Library (ACM), da Institute of Electrical and Electronics Engineers (IEEE) e da ScienceDirect (SD), Revista Brasileira de informática na Educação (RBIE), Portal SBGames (SBG), Comissão Especial de informática na Educação (CBIE-WIE e CBIE-SBIE).

\subsection{Critérios de inclusão e exclusão}

Para [Kitchenham 2004], critérios de seleção dos estudos são utilizados para determinar quais estudos serão incluídos ou excluídos na revisão sistemática. A fim de reduzir a probabilidade de polarização, critérios de seleção devem ser decididos durante a definição do protocolo, embora possam ser refinados durante o processo de busca. Os critérios de seleção definidos para este estudo são apresentados a seguir:

- Critérios de inclusão: artigos publicados em um período de dez anos (de 2004 a 2014) e que abordavam temas sobre educação, saúde (com o uso de jogos pelos idoso) e jogo digital para idosos.

- Critérios de exclusão: artigos que abordavam especificamente temas sobre a usabilidade de jogos, a realidade virtual, a interfaces de jogos para idosos, a área da saúde (exceto quando abordava o uso de jogos pelos idoso), os jogos digitais para crianças e os jogos digitais para jovens.

\subsection{Definição e avaliação da qualidade pretendida}

Segundo [Kitchenham 2004] definir e avaliar a qualidade pretendida para os estudos primários está além dos critérios de inclusão e exclusão. As questões de qualidade ajudam a classificar detalhes após a inclusão, a orientar recomendações futuras e a interpretar 
melhor os resultados da pesquisa. A dificuldade está em definir "qualidade". Contudo, as orientações de [Khan and Kleijnen 2001] sugerem que a qualidade refere-se à medida que o estudo minimiza viés e maximiza a validade interna e externa.

Os artigos que passaram pelos critérios de inclusão e exclusão são submetidos às questões de qualidade. As questões e os fatores de qualidade para este estudo podem ser vistos na Tabela 1. Os fatores de qualidade, definidos com base nas questões de pesquisa, são os seguintes: T (totalmente); P (Parcialmente); N (Não). Seguindo as diretrizes propostas por [Kitchenham 2004], a pontuação dos artigos ficou assim definida: $\mathrm{T}=1, \mathrm{P}=$ $0.5, \mathrm{~N}=0$.

Tabela 1. Critérios para a qualidade pretendida

\begin{tabular}{|l|l|l|l|}
\hline CRITÉRIO (C): & $\mathrm{T}$ & $\mathrm{P}$ & $\mathrm{N}$ \\
\hline $\begin{array}{l}\text { C1. A pesquisa abordou temas relevantes sobre jogos } \\
\text { educacionais digitais para idosos? }\end{array}$ & 1 & 0,5 & 0 \\
\hline C2. A pesquisa apresentou detalhes sobre o método utilizado? & 1 & 0,5 & 0 \\
\hline C3. Os dados coletados foram apresentados e descritos adequadamente? & 1 & 0,5 & 0 \\
\hline Total & 3 & 1,5 & 0 \\
\hline
\end{tabular}

\subsection{Avaliação das respostas às questões de pesquisa}

Ainda como fator de qualidade, verificou-se simultaneamente se os artigos respondiam às questões iniciais da pesquisa (RQ1, RQ2 e RQ3). Para isso, definiram-se os fatores de qualidade das respostas assim: T (respondeu satisfatoriamente); P (respondeu parcialmente); N (Não respondeu). Seguindo as diretrizes propostas por [Kitchenham 2004], a pontuação dos artigos, quanto às respostas às RQs, ficou assim definida: $\mathrm{T}=1, \mathrm{P}=0.5$, $\mathrm{N}=0$.

\subsection{Definição dos filtros da pesquisa}

Foram definidos quatro filtros para realizar a busca e a extração dos dados. O filtro 1 (principal) foi baseado na string de busca. Segundo [Kitchenham 2004], string de busca é uma sequência de caracteres, com os termos principais, adequadamente derivados das questões de pesquisa. A string final foi construída usando os operadores lógicos AND e $O R$ e foi adaptada para as regras das bases. Com a string de busca efetuou-se a pesquisa nas bases e selecionaram-se os estudos primários. A string final foi definida após os testes preliminares nas bases e pode ser vista a seguir:

\section{(educational OR learning OR training) AND (elderly or "old people") AND (game)}

O filtro 2 foi realizado a partir da leitura do título dos artigos, o filtro 3 a partir da leitura do resumo e o filtro 4 a partir da leitura diagonal (leitura rápida) dos estudos primários. Após cada busca, os artigos retornados foram organizados utilizando o software Mendeley ${ }^{1}$, que é um gerenciador de referências livre.

\footnotetext{
${ }^{1}$ https://www.mendeley.com
} 
CBIE-LACLO 2015

Anais do XXVI Simpósio Brasileiro de Informática na Educação (SBIE 2015)

\subsection{Síntese dos dados}

Os dados tabulados e apresentados foram os seguintes: a quantidade de estudos selecionados em cada uma das bases de pesquisa após a aplicação do filtro 1 (string); a quantidade de estudos selecionados após a aplicação dos filtros 2 (título), 3 (resumo) e 4 (leitura diagonal); a identificação, a base pesquisada, os autores, o título e o ano de publicação dos 18 artigos selecionados após a aplicação dos filtros; a avaliação da qualidade pretendida dos 18 artigos; a avaliação sobre as respostas encontradas nos artigos às questões de pesquisa.

\section{Fase 2 - Execução}

A partir dos resultados obtidos pelo filtro 1, as fontes RBIE, CBIE - WIE e CBIE - SBIE foram desconsideradas, por não terem retornado artigos que abordem o tema. Após aplicar o filtro 2, juntamente com os critérios e inclusão e exclusão, houve uma redução de 95\% dos artigos pré-selecionados. Após o filtro 3, houve uma redução de aproximadamente $74 \%$ e após o filtro 4, uma redução de aproximadamente $70 \%$. Os resultados da pesquisa foram tabulados e são apresentados nas Tabela 2.

Tabela 2. Coleta dos dados utilizando os filtros

\begin{tabular}{|l|c|c|c|c|c|}
\hline \multirow{2}{*}{ Fonte: } & \multirow{2}{*}{ Sigla } & \multicolumn{4}{c|}{ Filtro } \\
\cline { 3 - 6 } & & String & Título & Resumo & $\begin{array}{c}\text { Leitura } \\
\text { diagonal }\end{array}$ \\
\hline ACM Digital Library & ACM & 713 & 78 & 34 & 12 \\
\hline $\begin{array}{l}\text { Anais do Workshop de } \\
\text { Informática na Escola }\end{array}$ & CBIE-WIE & 0 & 0 & 0 & 0 \\
\hline $\begin{array}{l}\text { Anais do Simp. Brasileiro } \\
\text { de Informática na Educação }\end{array}$ & CBIE-SBIE & 0 & 0 & 0 & 0 \\
\hline IEEE Xplore & IEEE & 224 & 39 & 15 & 4 \\
\hline Portal SBGames & SBG & 610 & 40 & 7 & 2 \\
\hline $\begin{array}{l}\text { Revista Brasileira de } \\
\text { Informática na Educação }\end{array}$ & RBIE & 0 & 0 & 0 & 0 \\
\hline Science Direct & SD & 1590 & 13 & 6 & 2 \\
\hline \multicolumn{1}{|c|}{ TOTAL } & & $\mathbf{3 1 3 7}$ & $\mathbf{1 7 0}$ & $\mathbf{6 2}$ & $\mathbf{1 8}$ \\
\hline
\end{tabular}

Após a aplicação dos filtros foram selecionados 18 artigos, sendo 12 da ACM, quatro da IEEE Xplore e dois da SBG. Os artigos foram identificados pelas letras iniciais das fontes pesquisadas, sendo respectivamente A, I e $\mathrm{S}$ e são apresentados na Tabela 3. 
CBIE-LACLO 2015

Anais do XXVI Simpósio Brasileiro de Informática na Educação (SBIE 2015)

Tabela 3. Identificação dos artigos

\begin{tabular}{|c|c|c|c|c|}
\hline ID: & Base: & $\begin{array}{c}\text { Autor: } \\
\end{array}$ & $\begin{array}{c}\text { Título: } \\
\end{array}$ & ANO \\
\hline A1 & ACM & [Foukarakis et al. 2011] & An Adaptable Card Game for Older Users & 2011 \\
\hline $\mathbf{A 2}$ & $\overline{\mathrm{ACM}}$ & [Cheong et al. 2011] & Avatar: A Virtual Face for the Elderly & 2011 \\
\hline A3 & ACM & [Rice et al. 2012] & $\begin{array}{l}\text { Co-creating Games through Intergenerational } \\
\text { Design Workshops }\end{array}$ & 2012 \\
\hline A4 & $\mathrm{ACM}$ & [Gerling et al. 2011] & $\begin{array}{l}\text { Designing and Evaluating Digital Games for } \\
\text { Frail Elderly Persons }\end{array}$ & 2011 \\
\hline A5 & $\mathrm{ACM}$ & [Al Mahmud et al. 2008] & $\begin{array}{l}\text { Designing and Evaluating the Tabletop Game } \\
\text { Experience for Senior Citizens }\end{array}$ & 2008 \\
\hline A6 & $\mathrm{ACM}$ & [De Schutter and Vanden 2010] & $\begin{array}{l}\text { Designing Meaningful Play within the } \\
\text { Psycho-Social Context of Older Adults }\end{array}$ & 2010 \\
\hline A7 & $\mathrm{ACM}$ & [Pedell et al. 2013] & $\begin{array}{l}\text { Engaging Older Adults in Activity Group } \\
\text { Settings Playing Games on Touch Tablets }\end{array}$ & 2013 \\
\hline A8 & $\mathrm{ACM}$ & [Chu Yew Yee et al. 2010] & $\begin{array}{l}\text { Investigating Narrative in Mobile Games } \\
\text { for Seniors }\end{array}$ & 2010 \\
\hline A9 & $\mathrm{ACM}$ & [Brown 2012] & $\begin{array}{l}\text { Let!s Play: Understanding the Role and Meaning } \\
\text { of Digital Games in the Lives of Older Adults }\end{array}$ & 2012 \\
\hline A10 & ACM & [Gajadhar et al. 2010] & $\begin{array}{l}\text { Out of Sight, out of Mind: Co-Player Effects on } \\
\text { Seniors' Player Experience }\end{array}$ & 2010 \\
\hline A11 & $\mathrm{ACM}$ & [Shim et al. 2010] & $\begin{array}{l}\text { TableTalk Poker: An Online Social Gaming } \\
\text { Environment for Seniors }\end{array}$ & 2010 \\
\hline A12 & ACM & [Mubin et al. 2008] & $\begin{array}{l}\text { Walk } 2 \text { Win: Towards Designing a Mobile } \\
\text { Game for Elderly's Social Engagement }\end{array}$ & 2008 \\
\hline $\mathbf{I 1}$ & IEEE & [Ogomori et al. 2011] & $\begin{array}{l}\text { Requirements for a Cognitive Training Game } \\
\text { for Elderly or Disabled People }\end{array}$ & 2011 \\
\hline $\mathbf{I 2}$ & IEEE & [Bamidis et al. 2014] & $\begin{array}{l}\text { Using simulations and experiential learning } \\
\text { approaches to train carers of seniors }\end{array}$ & 2014 \\
\hline $\mathbf{I 3}$ & IEEE & [Chen et al. 2012b] & $\begin{array}{l}\text { Using Somatosensory Video Games to Promote } \\
\text { Quality of Life for the Elderly with Disabilities }\end{array}$ & 2012 \\
\hline I4 & IEEE & [Chiang et al. 2012] & $\begin{array}{l}\text { Using Xbox } 360 \text { Kinect Games on Enhancing } \\
\text { Visual Performance Skills on Institutionalized } \\
\text { Older Adults with Wheelchairs }\end{array}$ & 2012 \\
\hline S1 & SBG & [Santos et al. 2014] & $\begin{array}{l}\text { Casual mobile games for the elderly: a } \\
\text { usability study }\end{array}$ & 2014 \\
\hline $\mathbf{S 2}$ & SBG & [Carvalho 2012] & $\begin{array}{l}\text { Motivational Factors for Mobile Serious } \\
\text { Games for Elderly Users }\end{array}$ & 2012 \\
\hline
\end{tabular}

\subsection{Avaliação dos artigos em relação às respostas para as perguntas da pesquisa}

Os resultados da avaliação da qualidade dos dados, seguindo os critérios explicados na Tabela 1, são apresentados na Tabela 4. Os resultados da avaliação dos artigos em relação às respostas para as questões da pesquisa são apresentados na Tabela 5. Os artigos que alcançaram a pontuação menor que um nesta etapa foram desconsiderados. 
Tabela 4. Pontuação dos artigos, quanto à qualidade, após a aplicação dos filtros

\begin{tabular}{|r|r|r|r|r|r|}
\hline ID: & Q1: & Q2: & Q3: & ALCANÇADO: & PRETENDIDO: \\
\hline A1 & 1 & 1 & 0,5 & 2,5 & 3 \\
\hline A2 & 0 & 0,5 & 0,5 & 1 & 3 \\
\hline A3 & 0,5 & 0,5 & 0,5 & 1,5 & 3 \\
\hline A4 & 0,5 & 0,5 & 1 & 2 & 3 \\
\hline A5 & 0,5 & 0 & 0 & 0,5 & 3 \\
\hline A6 & 0,5 & 0,5 & 0 & 1,0 & 3 \\
\hline A7 & 0,5 & 0,5 & 0 & 1,0 & 3 \\
\hline A8 & 0 & 0 & 0,5 & 0,5 & 3 \\
\hline A9 & 0 & 0,5 & 0 & 0,5 & 3 \\
\hline A10 & 0,5 & 0,5 & 0,5 & 1,5 & 3 \\
\hline A11 & 0,5 & 0,5 & 0 & 1 & 3 \\
\hline A12 & 0,5 & 0,5 & 0,5 & 1,5 & 3 \\
\hline I1 & 0,5 & 0,5 & 0,5 & 1,5 & 3 \\
\hline I2 & 1 & 0 & 1 & 2 & 3 \\
\hline I3 & 0 & 0,5 & 0,5 & 1 & 3 \\
\hline I4 & 0,5 & 0 & 0 & 0,5 & 3 \\
\hline S1 & 1 & 1 & 1 & 3 & 3 \\
\hline S2 & 1 & 1 & 1 & 3 & \\
\hline
\end{tabular}

Tabela 5. Pontuação dos artigos, quanto às respostas das RQs, após a aplicação dos quatro filtros

\begin{tabular}{|r|r|r|r|r|r|}
\hline ID: & RQ1: & RQ2: & RQ3: & ALCANÇADO: & PRETENDIDO: \\
\hline A1 & 1 & 1 & 0,5 & 2,5 & 3 \\
\hline A3 & 0 & 0,5 & 0,5 & 1 & 3 \\
\hline A4 & 0,5 & 0,5 & 0 & 1 & 3 \\
\hline A6 & 0 & 1 & 0 & 1 & 3 \\
\hline A7 & 0 & 0,5 & 0,5 & 1 & 3 \\
\hline A10 & 0,5 & 0 & 0,5 & 1 & 3 \\
\hline A12 & 0,5 & 0 & 0,5 & 1 & 3 \\
\hline I1 & 0,5 & 0,5 & 0,5 & 1,5 & 3 \\
\hline I2 & 0 & 0,5 & 1 & 1,5 & 3 \\
\hline I3 & 0 & 0,5 & 0,5 & 1 & 3 \\
\hline S1 & 1 & 1 & 1 & 3 & 3 \\
\hline S2 & 1 & 1 & 1 & 3 & 3 \\
\hline
\end{tabular}

\section{Fase 3 - Relatório}

O presente relatório seguiu o modelo apresentado por [Kitchenham 2004]. Nesta seção são apresentadas as respostas encontradas nos artigos selecionados para as três questões de pesquisa (RQs). Das fontes pesquisadas, identificaram-se 12 estudos relevantes que foram apresentados na Tabela 3. Estes estudos tiveram suas qualidades, em relação às respostas para as perguntas de pesquisa (RQs), definidas conforme apresentado na Tabela 5, após a aplicação dos quatro filtros. Foram utilizadas as mesmas siglas adotadas para identificar os artigos citados na Tabela 3 . 


\subsection{RQ1. Quais as limitações cognitivas a serem consideradas no desenvolvimento de jogos digitais educacionais para idosos?}

Em A1, o tema principal trata de um projeto para o desenvolvimento de um jogo digital para múltiplos jogadores, adaptativo e voltado para o idoso. Os autores desse artigo abordam o problema das limitações cognitivas dos idosos, como perda de memória, de atenção, de imaginação, de associação, de percepção e de raciocínio, que precisam ser consideradas durante o desenvolvimento de jogos digitais para idosos. Os autores também abordam o potencial das tecnologias da informação para atender às necessidades cognitivas e motivações apresentadas por este público, tais como a diversão, a distração, a socialização e o aprendizado, aspecto este também destacado em S2. Os autores de A1 e $\mathrm{S} 1$ defendem que os jogos digitais devem respeitar critérios de acessibilidade, usabilidade e jogabilidade para que possam promover a inclusão e interação social dos idosos. Quando o jogo não respeita esses critérios, os idosos não conseguirão utilizá-lo, devido às suas limitações físicas e cognitivas.

Sob um outro aspecto, os autores de I1 abordam a utilização de protótipos eletromecânicos para criar jogos para idosos. Contudo esses protótipos não ajudam a explorar todo o potencial computacional de um jogo digital e nem tampouco respeitam as limitações cognitivas decorrentes do avanço da idade. Por isso, o uso de smartphones e computadores também é visto de forma positiva pelos autores em A10.

\subsection{RQ2. Quais os temas de interesse e expectativas dos idosos com relação a jogos digitais educacionais?}

Em S2, os autores realizaram um levantamento para identificar as características que os jogos digitais para smartphones devem ter, para que os idosos sintam o prazer e o desejo de usá-los. Os interesses e as expectativas citadas foram, por exemplo: diversão, distração, aprendizado, interação social, sentir menos solidão. Outro ponto citado pelos autores em S2 é sobre a expectativa e interesse dos idosos pelo desenvolvimento de produtos e serviços que os mantenham atualizados, fornecendo-lhes meios de aprendizagem, de entretenimento, de interatividade e de se comunicarem com as outras pessoas.

Por sua vez, em A6 e em A7, os autores destacam que para projetar jogos que atendam aos interesses e às expectativas do público-alvo a que o jogo se destina é importante considerar as características culturais desse público, tendo em vista que essas características variam para diferentes grupos de pessoas e que as pessoas se identificam com o contexto no qual se sentem bem. Ressalta-se, ainda, que para desenvolver os jogos educacionais digitais para os idosos deve-se levar em consideração que o custo-benefício conforme abordado em I3 e em A4.

\subsection{RQ3. Quais as contribuições dos jogos digitais educacionais para o processo de aprendizagem dos idosos?}

Em I2, os autores destacam que os ambientes tridimensionais podem contribuir para a aprendizagem dos idosos. Eles defendem o aprendizado pelas simulações em ambientes virtuais tridimensionais com um conjunto de componentes educacionais e objetos de aprendizagem. O aprendizado do idoso, pelo uso de jogos de simulação virtual, tridimensionais ou não, possui um grande potencial a ser pesquisado.

Os autores de S1 abordam a usabilidade como um fator que pode facilitar ou dificultar o processo da aprendizagem do idoso por meio dos jogos digitais e destacam 
que o jogo digital educacional para o idoso deve contribuir para exercitar a mente, a criatividade e a diversão.

Já em S2 os autores destacam que o jogo deve ser projetado com características que motivem os idosos a jogarem, contribuindo assim para o desenvolvimento da aprendizagem combinada com o entretenimento.

Sob um outro enfoque, os autores de A12 e de A3 afirmam que o projeto e a avaliação de jogos digitais para os idosos precisam ter o foco na interação social. $O$ argumento utilizado foi o de que ao jogar e interagir com outros jogadores, muitos idosos exercitam o relacionamento social e, paralelamente, aproveitam o tempo de forma mais agradável, podendo também aprender diferentes temas de acordo com as propostas dos jogos e com as interações com outros jogadores.

\section{Conclusões e recomendações}

Este estudo teve como objetivo aplicar o método da revisão sistemática de literatura, determinar e qualificar os estudos existentes sobre jogos educacionais para idosos e responder as questões inciais da pesquisa.

Os resultados tabulados e discutidos permitiram concluir que o método da revisão sistemática de literatura confirmou a carência de publicações sobre jogos digitais educacionais para idosos e permitiu selecionar e qualificar estudos que ajudaram a responder parcialmente às questões de pesquisa. Como resultado dos artigos selecionados, identificaram-se possíveis limitações cognitivas, temas e contribuições dos jogos digitais educacionais para os idosos.

Espera-se, portanto, que os resultados apresentados contribuam para aumentar os projetos e a produção científica sobre jogos digitais educacionais para os idosos. Como trabalhos futuros sugerem-se pesquisas e desenvolvimento de jogos educacionais que considerem as limitações cognitivas dos idosos, bem como seus temas de interesse e expectativas. Além disso, é importante realizar um estudo sobre o efeito dos jogos educacionais digitais no processo de aprendizagem do público-alvo deste artigo.

\section{Agradecimentos}

Esta pesquisa recebeu apoio financeiro do CNPq (475311/2012-4) e da FAPEMIG.

\section{Referências}

Al Mahmud, A., Mubin, O., Shahid, S., and Martens, J.-B. (2008). Designing and e. the tabletop game experience for senior citizens. In Of the 5th NC on HCI.

Bamidis, P., Antoniou, P., and Sidiropoulos, E. (2014). Using simulations and el approaches to train careers of seniors. In Computer-Based M. S., 2014 IEEE 27th IS on.

Brown, J. A. (2012). Let's play: Understanding the role and meaning of d. games in the 1. of older adults. In $O f$ the ICFDG.

Carvalho, Roberta N.and Ishitani, L. (2012). Motivational factors for mobile serious games for elderly users. In Proceedings of SBGames 2012.

Chen, S.-T., Chiang, I., Liu, E. Z.-F., Chang, M., et al. (2012a). Effects of improvement on s. a.: Developing appropriate somatosensory video game i. for institutional-dwelling elderly with disabilities. Turkish Online Journal of Educational Technology. 
CBIE-LACLO 2015

Anais do XXVI Simpósio Brasileiro de Informática na Educação (SBIE 2015)

Chen, S.-T., Huang, Y.-G., and Chiang, I.-T. (2012b). Using somatosensory v. games to promote quality of life for the elderly with disabilities. In Digital Game and Intelligent Toy Enhanced Learning, IEEE Fourth IC on.

Cheong, W. L., Jung, Y., and Theng, Y.-L. (2011). Avatar: A virtual face for the elderly. In Of the 10th IC on VRC and Its Aplications in Industry.

Chiang, I.-T., Tsai, J.-C., and Chen, S.-T. (2012). Using xbox 360 kinect games on enhancing vps on institutionalized older adults with wheelchairs. In Digital Game and Intelligent Toy Enhanced Learning, EEE Fourth IC on.

Chu Yew Yee, S. L., Duh, H. B.-L., and Quek, F. (2010). Investigating n. in mobile games for seniors. In $O f$ the $C H F$ in $C S$.

De Schutter, B. and Vanden, A. V. (2010). Designing m. play within the psycho-social context of older adults. In $O$ f the $3 r d$ International $C F G$.

Foukarakis, M., Leonidis, A., Adami, I., Antona, M., and Stephanidis, C. (2011). An adaptable c. game for older users. In $O f$ the 4th IC on PTR to AE.

Gajadhar, B. J., Nap, H. H., de Kort, Y. A. W., and IJsselsteijn, W. A. (2010). Out of sight, out of mind: Co-player e. on seniors' player experience. In $O f$ the $3 r d I C F G$.

Gerling, K. M., Schulte, F. P., and Masuch, M. (2011). Designing and e. d. games for frail elderly persons. In $O f$ the 8 th IC on Advances in CETE.

IBGE (2014). Síntese de is: análise das condições de vida da população brasileira.

Khan, Khalid, S. t. R. G. G. J. S. A. J. and Kleijnen, J. e. (2001). Undertaking systematic review of r. on e. In $C R D$ 's.

Kitchenham, B. A. (2004). Procedures for undertaking systematic reviews. Technical report, Computer Science Department, Keele University.

Mubin, O., Shahid, S., and Al Mahmud, A. (2008). Walk 2 win: Towards d. a m. game for elderly's social engagement. In Of the 22Nd BHCIGA CPC, Volume 2.

Ogomori, K., Nagamachi, M., Ishihara, K., Ishihara, S., and Kohchi, M. (2011). Requirements for a ctg for ederly or dp. In Biometrics and Kansei Eng, IC.

Pedell, S., Beh, J., Mozuna, K., and Duong, S. (2013). Engaging older adults in a. group settings p. games on touch tablets. In $O f$ the 25 th ACHIC.

Rice, M., Cheong, Y. L., Ng, J., Chua, P. H., and Theng, Y.-L. (2012). Co-creating games through intergenerational design workshops. In $O f$ the DISC.

Santos, L. G. N. O., Ishitani, L., and Nobre, C. N. (2014). Uso de jogos casuais em celulares por idosos: um estudo de usabilidade. In Proceedings of SBGames 2014.

Shim, N., Baecker, R., Birnholtz, J., and Moffatt, K. (2010). Tabletalk poker: An online social gaming e. for seniors. In Of the IAC on the FGD and Technology.

United Nations (2013). Wpp - the 2012 revision, Volume I: Comprehensive Tables.

World Health Organization (2014). Definition of an older or elderly person. Disponível em: http://goo.gl/InUvCp. Acesso em: 20 nov 2014. 